\title{
Risk factors for poor outcome in community-onset Clostridium difficile infection
}

\author{
Eunyoung Lee ${ }^{1,2}$, Kyoung-Ho Song ${ }^{1,2^{*}}$ (D) Ji Yun Bae ${ }^{2}$, Doran Yoon ${ }^{2}$, Joo-Hee Hwang ${ }^{2}$, Pyoeng Gyun Choe ${ }^{2}$, \\ Wan Beom Park², Ji Hwan Bang ${ }^{2}$, Eu Suk Kim², ${ }^{1,2}$ Sang Won Park², Nam Joong Kim², Myoung-don Oh² \\ and Hong Bin Kim ${ }^{1,2}$
}

\begin{abstract}
Background: A substantial portion of Clostridium difficile infection (CDI) cases occur in communities, and community-onset CDI (CO-CDI) can lead to serious complications including mortality. This study aimed to identify the risk factors for a poor outcome in CO-CDI.

Methods: We performed a retrospective review of all inpatients with CDI, in a 1300-bed tertiary-care hospital in Korea, from 2008 through 2015. CO-CDI was defined as CDI occurring within $48 \mathrm{~h}$ of admission. Poor outcome was defined as follows: 1) all-cause 30-day mortality, 2) in-hospital mortality, or 3) surgery due to CDI.

Results: Of a total 1256 CDls occurring over 8 years, $152(12.1 \%)$ cases were classified as CO-CDI and 23 (15.1\%) had a poor outcome, including $22(14.5 \%)$ cases of mortality and 2 (1.3\%) cases of surgery. Patients with a poor outcome had a higher mean age than those without a poor outcome (75.8 vs. 69.6 years, $p=0.03$ ). The proportion of men and prior proton pump inhibitor (PPI) use were significantly higher in the poor outcome group (65.2\% vs. $41.9 \%, p=0.04 ; 39.1 \%$ vs. $17.6 \%, p=0.02$, respectively). Multivariate binary logistic model showed that PPI use and anemia (hemoglobin $<10 \mathrm{~g} / \mathrm{dL}$ ) at presentation were significantly associated with a poor outcome (adjusted odds ratio [aOR], 3.76; 95\% confidence interval [95Cl], 1.26-11.21, aOR, 4.67; 95Cl, 1.52-14.34, respectively).
\end{abstract}

Conclusions: Clinicians should not only be aware of the possibility of CDI in the community setting but also pay more attention to PPI-using elderly patients with anemia in consideration of a poor outcome.

Keywords: Clostridium difficile, Community-acquired infections, Anemia, Proton pump inhibitors, Mortality, Risk factors

\section{Background}

Clostridium difficile is an anaerobic spore-forming bacterium that is resistant to gastric acid; thus, it can stay in the intestinal tract and proliferate when the normal gut flora is disrupted under specific circumstances such as antibiotic agent usage. C. difficile infection (CDI) has been identified as a major cause of nosocomial diarrhea. CDI can show various manifestations, from mild

\footnotetext{
* Correspondence: khsongmd@gmail.com; khsongmd@snu.ac.kr 'Department of Internal Medicine, Seoul National University Bundang Hospital, 82 Gumi-ro 173 beon-gil, Bundang-gu, Seongnam 463-707, Republic of Korea

${ }^{2}$ Department of Internal Medicine, Seoul National University College of Medicine, Seoul, Republic of Korea
}

diarrhea to severe colitis or toxic megacolon, which can result in surgery or even mortality.

The incidence of CDI has been increasing over the last 10 to 20 years, and several outbreaks of CDI have occurred globally [1]. In the United States, costs associated with CDI exceed $\$ 1.1$ billion per year, even with conservative estimates [2]. Recent studies have reported that a substantial portion of CDI occurs in the community and its incidence has been increasing [3]. In a previous report, only $24.2 \%$ of CDI occurred during hospitalization in the United States [4]. An Australian study reported that community-onset CDI (CO-CDI) accounted for $29 \%$ of CDI [5]; in a Taiwanese report, CO-CDI accounted of $15 \%$ $\mathrm{CDI}$ when the percentage of community-acquired CDI 
and community-onset healthcare-associated CDI were combined [6]. Moreover, some CO-CDI resulted in poor outcomes in those reports $[5,6]$.

Age over 70 years, leukocytosis, hypoalbuminemia, increased creatinine, nasogastric tube feeding, and small bowel obstruction or ileus are known risk factors for severe $\mathrm{CDI}$ and are associated with a high mortality $[7,8]$. However, some reports have revealed that patients with CO-CDI show different characteristics compared to patients with traditional nosocomial infection [9]. It is possible that the manifestations of complicated CO-CDI differ from those of nosocomial infection; however, few studies have investigated the risk factors for complications in CO-CDI. Thus, this study aimed to evaluate the clinical characteristics of CO-CDI and to identify the risk factors for a poor outcome in CO-CDI.

\section{Methods}

\section{Study design and definitions}

We performed a retrospective medical review in Seoul National University Bundang Hospital, which is a 1300-bed tertiary-care hospital located in Seongnam, Gyeonggi-do, South Korea. A medical record review was performed for all adults ( $\geq 18$ years old) admitted from 2008 to 2015.

CDI was defined as a positive $C$. difficile toxin test and diarrhea or an equivocal $C$. difficile toxin test, diarrhea, and treatment for CDI. Diarrhea was defined as described in the medical records or as defecation exceeding 3 times in a day. ELISA was used to identify $C$. difficile toxin A or B (Gemini analyser, Stratec Biomedical GmbH, Birkenfeld, Germany), performed as per the manufacturer's instructions in a single microbiology laboratory. CO-CDI was defined as CDI occurring within $48 \mathrm{~h}$ of admission. We regarded patients who were recurrently admitted multiple times within 1 week as one episode on the first admission day. When CDI occurred more than two times during one admission, we regarded the latter case as nosocomial CDI, which was excluded from this review. Poor outcome was defined if one of the following events occurred: 1) all-cause 30-day mortality, 2) in-hospital mortality or 3) surgery due to CDI. CDI treatment was defined as the use of intravenous/ oral metronidazole, oral vancomycin, or both.

\section{Study measures}

We collected epidemiological information such as sex, age, admission date, medical history including cardiovascular disease, diabetes mellitus, renal disease, hepatic disease, malignancy, and previous CDI from the medical records. A recent event of previous CDI was defined as an event occurring within the most recent 3 months. We reviewed recent medication histories such as antibiotic agent and proton pump inhibitor (PPI) use for the previous 1 month. We collected data on clinical manifestations (vital signs such as blood pressure, body temperature, Glasgow coma scale, stool frequency $>10$ per day, presence of mucoid stool, duration of diarrhea before admission, abdominal pain, abdominal tenderness, intensive care unit [ICU] admission), laboratory values (white blood cell [WBC] count, hemoglobin, creatinine, albumin, glucose), radiographic data (plain radiography, computed tomography $[\mathrm{CT}]$ ), and endoscopic results. Duration from admission to the treatment of CDI in days and the number of patients who needed to continue possible offending antimicrobial agents were reviewed. Laboratory data within $24 \mathrm{~h}$ from admission were collected and values with the greatest deviations from normal were collected if multiple values were available. In addition, medical images taken within 7 days of the $C$. difficile toxin test were reviewed.

\section{Statistical analysis}

The independent Student $t$-test or Mann-Whitney $U$-test was used to compare continuous variables between the poor and good outcome groups, depending on the normality of data. The chi-square test was used for categorical variables. Linear regression was used to evaluate trends of incidence. To evaluate independent factors for a poor outcome in CO-CDI, we designed two logistic regression models. The first model included significant demographic and clinical variables identified from univariate analysis. The second model included significant laboratory variables which were identified from univariate analysis and are known to be associated with severity in previous studies. We separated the variables into two models rather than combining all of them into one model. This was because a small study population and a large number of variables and covariates with potential collinearity might have affected the power of the study and the statistical relevance of the results. Two-sided tests were performed and $P$-values of $<0.05$ were considered statistically significant. The incidence rate was calculated for 10,000 admissions. All statistical analyses were performed with SAS for Windows, version 9.4 (SAS Institute, Inc., Cary, NC, USA).

\section{Results}

\section{Characteristics of the population}

During the 8-year study period, a total of 1256 CDI episodes were identified. Of these, 152 were classified as CO-CDI. Although the number of cases of CDI increased annually, the number of $C$. difficile toxin testing was also increased from 172 CDI cases/861 tests in 2008 to 256 CDI cases/2072 tests in 2015 (Additional file 1: Figure S1). However, there was no significant trend in the CDI incidence rate after the conversion 10,000 
admission rates per month $(p=0.36$, overall 32.75 per 10,000 admissions per month). There was no particular trend in the CO-CDI incidence rate $(p=0.51$, overall 3.64 per 10,000 admissions per month). No significant trend was observed in the incidence rate of complicated CO-CDI $(p=0.84$, overall 0.55 per 10,000 admissions per month).

The mean patient age was 70.66 (SD 14.89), with a slight female predominance $(54.6 \%)$ (Additional file 1: Table S1). There were 37 (24.3\%) cases from the long-term care facility. The mean Charlson's comorbidity index was 2.09 (SD 1.90) and there were 23 (15.0\%) cases with previous CDI; 119 (79.3\%) cases had a history of antibiotic agent usage within the previous 1 month. Fifteen (9.9\%) cases did not have an antibiotic or admission history for 3 months. Thirty-one (21.0\%) cases had a history of prior PPI use. There were $42(27.6 \%)$ cases with shock at the initial presentation. Eleven (7.2\%) cases required ICU admission.

We identified the prior use of antibiotic agents within 1 month in 110 (71.9\%) cases of CO-CDI. The antibiotic classification was ascertained in 95 (62.5\%) cases. The most frequently used antibiotic agent was cephalosporin and it was used for $48(50.5 \%)$ cases. Following, in the order of frequency, are fluoroquinolone in 37 (39.0\%), $\beta$-lactam $+\beta$-lactamase inhibitor in $19(20.0 \%)$, metronidazole in 9 (9.5\%), carbapenem in $8(8.4 \%)$ cases. Clindamycin was used in $2(2.1 \%)$ cases.

\section{Risk factors for a poor outcome in CO-CDI}

There were $23(15.1 \%)$ cases with a poor outcome. Of these, there were 22 (14.5\%) cases of mortality: 19 (12.5\%) during the admission period and 14 (9.2\%) within 30 days from admission. There were 2 cases of surgery, and of these, mortality occurred during admission in one case.

The mean age of patients and the proportion of men in the poor outcome group was higher than that in the good outcome group (75.8 vs. 69.6 years, $p=0.01 ; 65.2 \%$ vs. $41.9 \%, p=0.04$, respectively; Additional file 1: Table $\mathrm{S} 1)$. The mean heart rate within $24 \mathrm{~h}$ after admission was significantly higher in the poor outcome group (109.80 vs. 98.1 beats per $\min , p=0.01$ ); however, there was no significant difference in the proportion of septic shock or mean body temperature between the two groups. The rate of prior PPI use was higher in the poor outcome group $(39.1 \%$ vs. $17.6 \%, p=0.02)$. The rate of prior antibiotic agent use was not significantly different between the two groups $(73.9 \%$ vs. $80.3 \%, p=0.49)$. When analyzed by the classification of possible offending antibiotic agents, penicillin derivatives were more frequently used in the poor outcome group $(40.0 \%$ vs. $16.3 \%, p=0.03$; Additional file 1: Table S2). Clinical manifestations including mucoid stool, proportion of stool frequency exceeding 10 times, duration of symptoms, and abdominal pain or tenderness were not significantly different between the two groups. Laboratory values at presentation showed that the mean WBC count was higher in the poor outcome group, but this finding was not statistically significant. The mean hemoglobin level was marginally lower in the poor outcome group (10.42 g/dL vs. $11.30 \mathrm{~g} / \mathrm{dL}, p=0.06)$, and the mean bicarbonate and albumin levels were significantly lower in the poor outcome group than in the good outcome group $(19.52 \mathrm{mmol} / \mathrm{L}$ vs. $21.84 \mathrm{mmol} / \mathrm{L}$, $p=0.02 ; \quad 2.63 \mathrm{~g} / \mathrm{dL}$ vs. $3.21 \mathrm{~g} / \mathrm{dL}, \quad p=0.0001$, respectively).

Multivariate binary logistic analysis revealed that a history of PPI use was independently associated with a poor outcome (adjusted odds ratio [aOR], 3.88; 95\% confidence interval [95CI], 1.310-11.518). In addition, anemia of hemoglobin under $10 \mathrm{~g} / \mathrm{dL}$ was associated with a poor outcome (aOR, 3.55; 95CI, 1.230-10.219). Men were marginally associated with the increased risk of a poor outcome in CO-CDI (aOR, 2.74; 95CI, 0.998-7.525; Table 1).

\section{Discussion}

This study analyzed 152 CO-CDI episodes from a total of 1368 CDI cases, giving the proportion of $12.1 \%$. A substantial proportion (15.1\%) of CO-CDI cases underwent surgery and/or expired. Prior PPI use and anemia under hemoglobin $10 \mathrm{~g} / \mathrm{dL}$ were associated poor outcomes in multivariate analyses.

The prevalence of CO-CDI has been reported to range between 10 and $42 \%$ in various countries $[5,6,10]$. We observed that CO-CDI comprised $12.1 \%$ of CDI and this result is comparable with these previous studies. In this study, in-hospital mortality and 30-day mortality were 12.5 and $9.2 \%$, respectively. These rates are similar to findings in previous CO-CDI reports and are also comparable with the mortality in nosocomial CDI [6]. The factors associated with a poor outcome in CO-CDI were similar to those noted in previous studies in the univariate analysis but were slightly different in the multivariate analysis. Male sex and old age, similar to that in our study, have been identified as predictive risk factors for severe CDI in previous reports [7]. Elderly patients over 70 years comprised $62.5 \%$ of CO-CDI patients in our study. We confirmed that CO-CDI occurred more in the elderly and that they are more prone to have a poor outcome. In agreement with a previous report by Khanna et al. [10] which showed that CO-CDI in relatively young patients may follow a milder clinical course, we demonstrated that older patients with CO-CDI still had a higher risk of a poor outcome.

To the best of our knowledge, no previous report has attempted to clarify the risk factors for severe outcomes 
Table 1 Risk factors for poor outcome in community-onset Clostridium difficile infection in multivariate logistic regression analyses

\begin{tabular}{llll}
\hline Variable & Odds ratio & $95 \% \mathrm{Cl}$ & $P$ \\
\hline Age $>70$ y & 1.47 & $0.468-4.627$ & 0.51 \\
Male & 2.74 & $0.998-7.525$ & 0.05 \\
Long-term care facility residence & 0.98 & $0.287-3.354$ & 0.98 \\
Glasgow coma scale $\leq 12$ & 2.06 & $0.589-7.185$ & 0.26 \\
Presentation with septic shock & 0.84 & $0.256-2.742$ & 0.77 \\
Heart rate $\geq 100 /$ min & 2.81 & $0.972-8.102$ & 0.06 \\
Prior proton pump inhibitor use within 1 month & 3.88 & $1.310-11.518$ \\
ICU admission & 2.71 & $0.599-12.257$ \\
Max leukocyte count $>20,000 / \mathrm{mL}$ & 2.78 & $0.897-8.684$ & 0.01 \\
Min hemoglobin level $<10 \mathrm{~g} / \mathrm{dL}$ & 3.55 & $1.230-10.219$ \\
Min bicarbonate level $<20 \mathrm{mmol} / \mathrm{L}$ & 2.16 & $0.736-6.356$ \\
Max glucose level $>150 \mathrm{mg} / \mathrm{dL}$ & 0.83 & $0.259-2.623$ \\
Max creatinine level $>2 \mathrm{mg} / \mathrm{dL}$ & 0.54 & $0.160-1.841$ \\
Min albumin level $<2.5 \mathrm{~g} / \mathrm{dL}$ & 1.21 & $0.373-3.928$ \\
\hline
\end{tabular}

ICU Intensive care unit, Max maximum, Min Minimum, $\mathrm{Cl}$, confidence interval

Poor outcome was defined if at least one of following event was occurred: 1) all-cause 30-day mortality, 2) in-hospital mortality, or 3) surgery due to $C$. difficile infection

Clinical variables $(n-152)$ and laboratory variables $(n=151)$ were analyzed in two separated models in consideration of potential collinearity Laboratory results were the most deviated value from normal range within $24 \mathrm{~h}$ after admission

in CO-CDI. This study demonstrated that PPI use is associated with a poor outcome in CO-CDI, similar to that in nosocomial CDI. PPI is known to be associated with the development $[9,11]$, severity [12], and recurrence [8] of CDI, and has recently been reported to be associated with the development of CO-CDI [13]. In a pharmacoepidemiologic cohort study, Howell et al. [14] reported that iatrogenic gastric acid suppression increases CDI development with a dose-response effect, suggesting a causal relationship. In an in vitro study, Jump et al. [15] reported the prolonged survival of $C$. difficile in the gastric contents of PPI-using patients with elevated $\mathrm{pH}(\mathrm{pH}>5)$. Another study reported that exposure to PPI promoted toxin gene expression of $C$. difficile [16]. Furthermore, an experimental study with mice demonstrated that toxin A and B titers and myeloperoxidase activity, which is a marker for inflammatory activity was elevated in both antibiotic and proton pump inhibitor-receiving groups [17]. This finding suggests that PPIs should be appropriately used.

Anemia reflects the general condition of a patient, such as nutritional status (e.g. iron, vitamin deficiency), as well as underlying chronic diseases. However, we could not find any previous report regarding a direct association of anemia with human CDI. In a study, an association between anemia and colitis was noted in a mouse acute colitis model [18]. Anemia is also influenced by the pro-inflammatory cytokine-mediated inhibition of erythropoiesis [19]. It is possible that anemia in patients at the initial presentation of CDI might represent the severity of CDI associated with relative ischemia in an inflammatory condition of the colon. Thus, we thought that anemia could be a significant factor for poor outcomes in CO-CDI, as a representative of an underlying condition and severity of acute illness.

Regarding possible offending antibiotic agents, cephalosporin was used most frequently, followed by fluoroquinolone, and $\beta$-lactam $+\beta$-lactamase inhibitor in a descending order. Conventionally, clindamycin is a known risk factor for nosocomial CDI and CO-CDI [20]. In this report, only 2 patients were administered clindamycin before admission. One reason for this is that clinicians may have avoided prescribing clindamycin because of the associated risk of CDI. Additionally, broad-spectrum antibiotics such as $\beta$-lactam $+\beta$-lactamase inhibitor or carbapenem are being used increasingly, decreasing the need for extra anaerobic bacterial coverage [21]. Except for clindamycin, three dominant antibiotic classes in this study were noted among previously reported drugs associated with CO-CDI risk in a meta-analysis [22].

This study has some limitations. The first limitation of this study includes its single-center retrospective study design. Cases of CDI could have been missed if primary physicians did not suspect CDI and did not test for the C. difficile toxin in patients from the community with relatively mild diarrhea, who did not have a history of antibiotic use. Thus, the CDI prevalence rate could have been underestimated. This assumption might explain the older age and higher proportion of febrile patients in this study compared to a previous community-based study [23]. However, we believe that our study included sufficient cases of severe CO-CDI and is valuable in this 
regard. Second, we did not investigate whether microbiological characteristics (e.g., ribotype) are associated with complicated CO-CDI. In a nationwide study from 12 hospitals in Korea, a major strain of nosocomial CDI was ribotype 017 [24]. There was limited data on the $C$. difficile strain in the case of CO-CDI in Korea, and we did not find any report on the relationship between ribotype and severity. Although the hypervirulent strain of ribotype 027 was described for the first time in Korea in 2009 [25], it does not appear to be epidemic or endemic in Korea yet. Considering previous reports on the prevalence rate in Korea, it does not appear to be an epidemic of a hypervirulent strain based on the prevalence rate compared to a previous study in Korea [26].

\section{Conclusions}

In this study, a substantial proportion of CDI occurred in the community. Clinicians should suspect CO-CDI in patients with diarrhea, abdominal pain, abdominal tenderness, and fever, who have a history of antibiotic use. In particular, patients with anemia at presentation and who use PPI need additional attention considering the identification of anemia and PPI use as risk factors for a poor outcome.

\section{Additional file}

Additional file 1: Table S1. Clinical characteristics of patients with community-onset Clostridium difficile infection. Table S2. Possible offending antibiotic agents in patients with community-onset Clostridium difficile infection. Figure S1. Incidence of C. difficile infection from 2008 through 2015 (DOCX 183 kb)

\section{Abbreviations}

95Cl: 95\% confidence interval; aOR: Adjusted odds ratio; CDI: Clostridium difficile infection; CO-CDI: Community-onset Clostridium difficile infection; CT: Computed tomography; PPI: Proton pump inhibitor; WBC: White blood cell

\section{Acknowledgements}

We express our gratitude to Ji Young Kwak for her active participation in the screening and case recording of CO-CDI in Seoul National University Bundang Hospital.

\section{Funding}

This work was supported by the SNUBH Research Fund [Grant no. 02-2017031]. The funding source had no role in the design of this study and did not influence the execution, analyses, interpretation of the data, or decision to submit results.

\section{Availability of data and materials}

All data generated or analyzed during this study are included in this published article.

\section{Authors' contributions}

Study design: EL, KHS, ESK, HBK. Data collection and analysis: EL, KHS, JYB, DY, JHH, PGC, WBP, JHB, ESK, SWP, NJK, MO, HBK. Statistical analysis: EL, KHS. Manuscript writing and critical revision: EL, KHS, ESK, NJK, MO, HBK. Manuscript review: All authors read and approved the final manuscript.

\section{Ethics approval and consent to participate}

This study was approved by the institutional review board of Seoul National University Bundang Hospital (B-1703-385-107) according to the Helsink Declaration. The need for informed consent was waived by the board.

\section{Competing interests}

The authors declare that they have no competing interests.

\section{Publisher's Note}

Springer Nature remains neutral with regard to jurisdictional claims in published maps and institutional affiliations.

Received: 5 January 2018 Accepted: 4 June 2018

Published online: 15 June 2018

\section{References}

1. Khanna S, Pardi DS. The growing incidence and severity of Clostridium difficile infection in inpatient and outpatient settings. Expert Rev Gastroenterol Hepatol. 2010:4:409-16.

2. Kyne L, Hamel MB, Polavaram R, Kelly CP. Health care costs and mortality associated with nosocomial diarrhea due to Clostridium difficile. Clin Infect Dis. 2002;34:346-53.

3. Evans CT, Safdar N. Current trends in the epidemiology and outcomes of Clostridium difficile infection. Clin Infect Dis. 2015;60:S66-71.

4. Lessa FC, Mu Y, Bamberg WM, Beldavs ZG, Dumyati GK, Dunn JR, et al. Burden of Clostridium difficile infection in the United States. N Engl J Med. 2015;372:825-34.

5. Foster N, Collins D, Ditchburn S, Duncan C, Schalkwyk J, Golledge C, et al. Epidemiology of Clostridium difficile infection in two tertiary-care hospitals in Perth, Western Australia: a cross-sectional study. New Microbes New Infect. 2014;2:64-71.

6. Tsai C-S, Hung Y-P, Lee J-C, Lee N-Y, Chen P-L, Syue L-S, et al. Communityonset Clostridium difficile infection at a tertiary medical center in southern Taiwan, 2007-2015. J Microbiol Immunol Infect. 2018;51:243-50.

7. Henrich TJ, Krakower D, Bitton A, Yokoe DS. Clinical risk factors for severe Clostridium difficile-associated disease. Emerg Infect Dis. 2009:15:415-22.

8. Moshkowitz M, Ben-Baruch E, Kline Z, Shimoni Z, Niven M, Konikoff F. Risk factors for severity and relapse of pseudomembranous colitis in an elderly population. Color Dis. 2007:9:173-7.

9. Pituch $\mathrm{H}$. Clostridium difficile is no longer just a nosocomial infection or an infection of adults. Int J Antimicrob Agents. 2009;33:S42-S5.

10. Khanna S, Pardi DS, Aronson SL, Kammer PP, Orenstein R, St Sauver JL, et al. The epidemiology of community-acquired Clostridium difficile infection: a population-based study. Am J Gastroenterol. 2012;107:89-95.

11. Janarthanan S, Ditah I, Adler DG, Ehrinpreis MN. Clostridium difficileassociated diarrhea and proton pump inhibitor therapy: a meta-analysis. Am J Gastroenterol. 2012:107:1001-10.

12. Lewis PO, Litchfield JM, Tharp JL, Garcia RM, Pourmorteza M, Reddy CM. Risk and severity of hospital-acquired Clostridium difficile infection in patients taking proton pump inhibitors. Pharmacotherapy. 2016;36:986-93.

13. Dial S, Delaney J, Barkun AN, Suissa S. Use of gastric acid-suppressive agents and the risk of community-acquired Clostridium difficile-associated disease. JAMA. 2005;294:2989-95

14. Howell MD, Novack V, Grgurich P, Soulliard D, Novack L, Pencina M, et al. latrogenic gastric acid suppression and the risk of nosocomial Clostridium difficile infection. Arch Intern Med. 2010;170:784-90.

15. Jump RL, Pultz MJ, Donskey CJ. Vegetative Clostridium difficile survives in room air on moist surfaces and in gastric contents with reduced acidity: a potential mechanism to explain the association between proton pump inhibitors and C. Difficile-associated diarrhea? Antimicrob Agents Chemother. 2007;51:2883-7.

16. Stewart DB, Hegarty JP. Correlation between virulence gene expression and proton pump inhibitors and ambient $\mathrm{pH}$ in Clostridium difficile: results of an in vitro study. J Med Microbiol. 2013;62:1517-23.

17. Kaur S, Vaishnavi C, Prasad KK, Ray P, Kochhar R. Comparative role of antibiotic and proton pump inhibitor in experimental Clostridium difficile infection in mice. Microbiol Immunol. 2007:51:1209-14.

18. Carter PR, Watts MN, Kosloski-Davidson M, Prasai K, Grisham MB, Harris NR. Iron status, anemia, and plasma erythropoietin levels in acute and chronic mouse models of colitis. Inflamm Bowel Dis. 2013;19:1260-5. 
19. Morceau F, Dicato M, Diederich M. Pro-inflammatory cytokine-mediated anemia: regarding molecular mechanisms of erythropoiesis. Mediat Inflamm 2009; https://doi.org/10.1155/2009/405016

20. Johnson S, Samore MH, Farrow KA, Killgore GE, Tenover FC, Lyras D, et al. Epidemics of diarrhea caused by a clindamycin-resistant strain of Clostridium difficile in four hospitals. N Engl J Med. 1999;341:1645-51.

21. Yoon YK, Park GC, An H, Chun BC, Sohn JW, Kim MJ. Trends of antibiotic consumption in Korea according to National Reimbursement Data (20082012): a population-based epidemiologic study. Medicine (Baltimore). 2015; 94:e2100.

22. Deshpande A, Pasupuleti V, Thota P, Pant C, Rolston DD, Sferra TJ, et al. Community-associated Clostridium difficile infection and antibiotics: a meta-analysis. J Antimicrob Chemother. 2013;68:1951-61.

23. Bauer $M$, Veenendaal $D$, Verhoef $L$, Bloembergen P, Van Dissel J, Kuijper E. Clinical and microbiological characteristics of community-onset Clostridium difficile infection in the Netherlands. Clin Microbiol Infect. 2009;15:1087-92.

24. Kim H, Jeong SH, Roh KH, Hong SG, Kim JW, Shin M-G, et al. Investigation of toxin gene diversity, molecular epidemiology, and antimicrobial resistance of Clostridium difficile isolated from 12 hospitals in South Korea. Korean J Lab Med. 2010;30:491-7.

25. Tae CH, Jung S, Song HJ, Kim S-E, Choi HJ, Lee M, et al. The first case of antibiotic-associated colitis by Clostridium difficile PCR ribotype 027 in Korea. J Korean Med Sci. 2009;24:520-4.

26. Kim J, Pai H, Seo MR, Kang JO. Epidemiology and clinical characteristics of Clostridium difficile infection in a Korean tertiary hospital. J Korean Med Sci. 2011;26:1258-64

Ready to submit your research? Choose BMC and benefit from:

- fast, convenient online submission

- thorough peer review by experienced researchers in your field

- rapid publication on acceptance

- support for research data, including large and complex data types

- gold Open Access which fosters wider collaboration and increased citations

- maximum visibility for your research: over $100 \mathrm{M}$ website views per year

At BMC, research is always in progress.

Learn more biomedcentral.com/submissions 